\title{
GENERALIZACIÓN DE LA INTEGRAL DE ORDEN ORDINARIO A ORDEN FRACCIONARIO
}

\author{
GENERALIZATION OF THE COMPREHENSIVE ORDINARY ORDER TO \\ FRACTIONAL ORDER
}

${ }^{1}$ Jhony Chávez Delgado, ${ }^{2}$ Luis Méndez Avalos, ${ }^{3}$ Luis Chávez Delgado

\begin{abstract}
RESUMEN
El propósito de este artículo es demostrar la generalización de la integral de orden entero de Newton - Leibniz al operador de integración de orden fraccionario de Riemann-Liouville sobre un intervalo cerrado. En tal sentido, se presenta la teoría básica de las diversas aproximaciones de la integral de orden ordinario, el uso de la función gamma y la fórmula de Cauchy; los cuales sirven de base para llegar a la definición del operador de integración fraccionario, a partir de la nésima integral iterado ordinario de una función definida recursivamente. Luego, se hace las demostraciones y ejemplificaciones de la linealidad del operador integral fraccionario, y las proposiciones de este operador fraccionario aplicado a la función potencia y logarítmica.
\end{abstract}

Palabras claves: Integral de una potencial, integral de un logaritmo, integración fraccionaria.

\begin{abstract}
It is the purpose, through this article, to demonstrate the generalization of the whole integral of Newton - Leibniz to the fractional order integration operator of Riemann-Liouville over a closed interval. In this sense, the basic theory of the different approximations of the integral of ordinary order, the use of the gamma function and the Cauchy formula are presented, which serve as the basis to arrive at the definition of the fractional integration operator from the $\mathrm{n}$-th ordinary iterated integral of a recursively defined function. Then, the demonstrations and exemplifications of the linearity of the fractional integral operator, and the propositions of this fractional operator applied to the power and logarithmic function are made.
\end{abstract}

Keywords: Integral of a potential, integral of a logarithm, fractional integration.

\section{INTRODUCCIÓN}

En el estudio del cálculo integral, los estudiantes universitarios de ciencias e ingeniería solucionan operadores integrales $I^{1} f, I^{2} f, I^{3} f, I^{4} f, \ldots$ de las funciones potencial y logarítmica, etc.; algunos, adicionalmente, reflexionan sobre si es necesario que el orden de la integral sea un número entero. Por ejemplo, se cuestionan sobre por qué los operadores de integración de la función logarítmica y potencia no han de ser $I^{1 / 2} f, I^{1 / 3} f \ldots$ Preguntas que se responden en el presente artículo.

El cálculo de la derivada y la integral de Newton Leibniz como rama del análisis matemático, a través del tiempo, ha sido estudiado por reconocidos matemáticos, quienes han contribuido al desarrollo de lo que hoy conocemos como cálculo fraccionario y que tiene interés en las órdenes de los operadores de derivación e integración. Entre ellos destacamos a Euler (1738), Liouville (1832) y Riemann (1876). Así mismo, Bertram (1975); Miller y Ross (1993); Samko, Kilbas y Marichev (1993); Bonilla, Kilbas y Trujillo (2003); Arafet, Domínguez y Chang (2008); Santanu (2011).

Uno de los propósitos de este artículo es establecer la generalización de la integral de orden ordinario de Newton-Leibniz a orden fraccionario de RiemannLiouville, utilizando como base la función gamma y la fórmula de Cauchy para llegar a definir la integral

\footnotetext{
Docente del Departamento de Matemática y Estadística. Universidad Nacional Jorge Basadre Grohmann. Tacna- Perú. E-mail: jhonychavez@yahoo.es

${ }^{1}$ Docente del Departamento de Matemática y Estadística. Universidad Nacional Jorge Basadre Grohmann. Tacna- Perú. E-mail: luismendezavalos@hotmail.com

${ }_{3}^{2}$ Docente del Programa de Formación General. Universidad César Vallejo. Chimbote-Perú. E-mail: 1achd_1908@hotmail.com 
fraccionaría. Además, se pretende demostrar que existe más de una manera de calcular la integral entera a orden fraccionario, usando un operador de integración.

Asimismo, la generalización de la integral de orden ordinario a orden fraccionario se justifica, debido principalmente a la gran importancia para el desarrollo del cálculo fraccionario; pues con la integral también se explicaría el uso de la derivada de orden fraccional. A pesar de la gran cantidad de publicaciones en este campo, aún se necesita formalizar y ordenar los conceptos, proposiciones y ejemplificaciones de la integral fraccionaria, para hacerla más atractiva y de fácil acceso a los investigadores de otras ciencias puras y aplicadas como Matemática, Física, Química, Biología, Economía...

Este artículo se enmarca en el campo de la integral fraccionaria y pretende ser una introducción al estudio de la derivación fraccionaria y a las ecuaciones diferenciales ordinarias de orden fraccionario asociado al operador de Riemann-Liouville y sus aplicaciones a la dinámica de una esfera inmersa en un fluido viscoso incomprensible. Procesos oscilatorios con amortiguación fraccionaria (Kilbas y Trujillo, 2003). Así como también, la ingeniería de control y el procesamiento de señales (Ortigueira, 2011).

Euler (1738) introdujo la función Gamma en el siglo XVIII. La interpretación más simple que podemos dar de esta función es que constituye la generalización de la factorial de todo número real, aunque el argumento de esta sea en general un número complejo. Sirvió de base para llegar a la definición de la integral fraccionaria.

\section{Definición (función Euleriana)}

Sea $0>x$. A la función integral $\Gamma$ definida por:

$$
\Gamma(x)=\int_{0}^{+\infty} e^{-t} t^{x-1} d t
$$

, se le denomina función Gamma de Euler.

\section{Propiedades de la función Gamma de Euler}

Sea $x>0$, entonces se verifica que

(a) $\Gamma(x+1)=x \Gamma(x), x>0$

(b) $\mathrm{Si} \mathrm{x}$ es un entero no negativo, entonces $\Gamma(x+1)=x$ !

(c) Si $x$ es un entero positivo, entonces $\Gamma(x+1 / 2)=\frac{(2 x) ! \sqrt{\pi}}{2^{2 x} x !}$. Bell (1968), y Levedev (1972).

\section{Definición (función beta)}

Sea $x>0$ e $y>0$.A la función integral $B$ definida por:

$$
B(x, y)=\int_{0}^{1} t^{x-1}(1-t)^{y-1} d t
$$

se le denomina función Beta. Chaudhry \& Zubair (2002).

\section{Propiedades de la función Beta}

Sea $x>0$ e $y>0$, entonces se verifica que
(a) $B(x, y)=\frac{\Gamma(x) \Gamma(y)}{\Gamma(x+y)}$
(b) $B(x, y)=B(y, x)$

Tabla 1. Valores de la función Gamma.

\begin{tabular}{lc} 
& $\Gamma(x)=\int_{0}^{+\infty} e^{-t} t^{x-1} d t, x>0$ \\
\hline$x$ & $\Gamma(x)$ \\
$1 / 2$ & $\sqrt{\pi}$ \\
$3 / 2$ & $\frac{1}{2} \sqrt{\pi}$ \\
$4 / 3$ & $0,8930 \ldots$ \\
$3 / 4$ & $1,2254 \ldots$ \\
$7 / 6$ & $0,9277 \ldots$ \\
$11 / 6$ & $0,9407 \ldots$
\end{tabular}

Fuente: Elaboración propia.

\section{Definición (función digamma)}

La derivada logarítmica de la función Gamma se denomina la función digamma y se define por:

$$
\psi(z)=\frac{\Gamma^{\prime}(z)}{\Gamma(z)}, \quad z>0
$$

También se conoce como la función psi $(\psi)$ o de Euler. Chaudhry \& Zubair (2002)

\section{Propiedades de la función digamma}

(a) $\psi(z+1)=\psi(z)+\frac{1}{z}, z>0$

(b) $\quad \psi(1)=\Gamma^{\prime}(1)=-\gamma$, donde $\gamma=0,577215664901532860606512090$...

es llamada la constante de Euler.

(c) Si $\alpha>-1$ y $\beta>-1$, entonces

$$
\Gamma(\mathrm{z})=\int_{0}^{1} \frac{\mathrm{x}^{\alpha} \cdot \mathrm{x}^{\beta}}{1-\mathrm{x}} \mathrm{dz}=\psi(\beta+1)-\psi(\alpha+1) .
$$

Chaudhry \& Zubair (2002). 
Tabla 2: Valores de la Función Digamma

$$
\begin{aligned}
& \psi(x)=\frac{\Gamma^{\prime}(x)}{\Gamma(x)}, x>0 \\
& \psi(1)=-\gamma \\
& \psi(n+1)=-\gamma+1+\frac{1}{2}+\frac{1}{3}+\cdots+\frac{1}{n}(n=0,1,2,3, \ldots) \\
& \psi\left(\frac{1}{2}\right)=-\gamma-2 \ln 2 \\
& \psi\left(\frac{1}{3}\right)=-\gamma-\frac{\pi}{2 \sqrt{3}}-\frac{3}{2} \ln 3 \\
& \psi\left(\frac{1}{4}\right)=-\gamma-\frac{\pi}{2}-3 \ln 2 \\
& \psi\left(\frac{2}{3}\right)=-\gamma+\frac{\pi}{2 \sqrt{3}}-\frac{3}{2} \ln 3
\end{aligned}
$$

Fuente: Chaudhry \& Zubair(2002)

\section{MÉTODOS}

En nuestro artículo es considerado material de estudio el operador lineal de integración aplicada a una función real $f$ sobre un intervalo finito $[a, b]:$ Sea $\alpha \in \mathbb{R}^{+}$. La integración fraccionaria de orden $\alpha$ de una función $\mathrm{f} \in$ $\mathrm{L}^{1}[\mathrm{a} ; \mathrm{b}]$, está dada por el operador lineal:

$$
{ }_{a} I_{x}^{a} f(x)=\frac{1}{\Gamma(\alpha)} \int_{a}^{x}(x-t){ }^{\alpha-1} f(t) d t, a \leq x \leq b, \alpha>0 .
$$

El método usado es axiomático-deductivo, el mismo que permite establecer un conjunto de reglas de razonamiento, de enunciados y postulados a partir de los cuales, y por reglas de inferencia del sistema, se derivan otros enunciados o proposiciones (Guardales, 2004).

\section{RESULTADOS}

\section{Operador de integración de orden fraccionario}

Sea $\alpha \in \mathbb{R}^{+}$y $f$ una función integrable en $L^{1}[a ; b]$. Entonces $\forall x \in[a ; b]$

$$
{ }_{a} I_{x}^{\alpha} f(x)=\frac{1}{\Gamma(\alpha)} \int_{a}^{x}(x-s)^{\alpha-1} f(s) d s
$$

Bonilla, Kilbas y Trujillo (2003)

Linealidad del operador de integración de orden fraccionario

Sea $f$ una función integrable en $\mathrm{f} \in \mathrm{L}^{1}[\mathrm{a}, \mathrm{b}]$. Luego, para $a \leq x \leq b y \quad \alpha \in \mathbb{R}^{+}$tenemos: (a) ${ }_{a} I_{x}^{\alpha} \lambda f(x)=\lambda_{a} I_{x}^{\alpha} f(x)$

(b) ${ }_{a} I_{x}^{\alpha}(f(x)+g(x))={ }_{a} I_{x}^{\alpha} f(x)+{ }_{a} I_{x}^{\alpha} g(x)$

(c) Sea $\alpha_{1}, \alpha_{2} \in \mathbb{R}^{+}$y f $\in \mathrm{L}^{1}[\mathrm{a}, \mathrm{b}]$

Entonces ${ }_{a} I_{x{ }_{a}}^{\alpha_{1}} I_{x}^{\alpha_{2}} f(x)={ }_{a} I_{x}^{\alpha_{2}} I_{x}^{\alpha_{1}} f(x)$

$=I_{x}^{\alpha_{1}+\alpha_{2}} f(x)$

se cumple casi para todo $x \in[a, b]$. Bonilla, Kilbas y Trujillo (2003)

Operador de Integración de orden fraccionario de una función potencial

Si $f(x)=(x-a)^{\beta}, \beta>0, x>a, \alpha>0$ entonces ${ }_{a} I_{x}^{\alpha}(x-a)^{\beta}=\frac{\Gamma(\beta+1)}{\Gamma(\alpha+\beta+1)}(x-a)^{\alpha+\beta}$

Miller \& Ross (1993)

\section{Operador de Integración de orden fraccionario de} una función logarítmica

Si $f(x)=\ln (x-a)$ tal que $x>a, \alpha>0$ entonces ${ }_{a} I_{x}^{\alpha} \ln (x-a)=\frac{(x-a)^{\alpha}}{\Gamma(\alpha+1)}[\ln (x-a)-\gamma-\psi(\alpha+1)]$ Miller \& Ross (1993)

\section{DISCUSIÓN}

La matemática en tanto ciencia eidética o formal - como dice Bunge- utiliza el método deductivo; es decir, mediante deducciones lógicas obtiene verdades o afirmaciones a partir de axiomas previamente formuladas.

El método deductivo es utilizado para justificar matemáticamente las condiciones suficientes de las propiedades de la integral de orden ordinario a orden fraccionario y el método inductivo es para contrastar el funcionamiento de dichas condiciones, utilizando ejemplos conocidos.

\section{Operador de Integración del orden fraccionario Proposición (Fórmula de Cauchy.A)}

Sea $n \in \mathbb{N}$ y $f \in \mathrm{L}^{1}[a, b] . L a$-ésima integral está dado por:

$$
{ }_{a} I_{x}^{n} f(x)=\frac{1}{\Gamma(n)} \int_{a}^{x}(x-t)^{n-1} f(t) d t, t>a, n \in \mathbb{N}
$$




\section{Demostración}

Sea $f \in \mathrm{L}^{1}[\mathrm{a}, \mathrm{b}]$.Definimos para $\mathrm{x} \in[\mathrm{a}, \mathrm{b}]$ la función $\mathrm{f}$ con límite de integración inferior $\mathrm{a}=0$

${ }_{0} I_{x}^{1} f(x)=\int_{0}^{x} f(t) d t$

Para dos integrales se tiene:

${ }_{0} I_{x}^{2} f(x)=\int_{0}^{x} \int_{0}^{x_{1}} f(t) d t d x_{1}$

y por el teorema de Fubini, podemos intercambiar el orden de integración en la integral doble (1.2):

$$
\begin{aligned}
& { }_{0} I_{x}^{2} f(x)=\int_{0}^{x} \int_{t}^{x} f(t) d x_{1} d t \\
& { }_{0} I_{x}^{2} f(x)=\int_{0}^{x} f(t) \int_{t}^{x} d x_{1} d t \\
& { }_{0} I_{x}^{2} f(x)=\int_{0}^{x} f(t)\left[x_{1}\right]_{t}^{x} d t \\
& { }_{0} I_{x}^{2} f(x)=\int_{0}^{x} f(t)[x-t] d t
\end{aligned}
$$

Ahora consideremos tres integrales:

$$
\begin{aligned}
& { }_{0} I_{x}^{3} f(x)=\int_{0}^{x} \int_{0}^{x_{1}} \int_{0}^{x_{2}} f(t) d t d x_{2} d x_{1} \\
& { }_{0} I_{x}^{3} f(x)=\int_{0}^{x} \int_{0}^{x_{1}} \int_{t}^{x_{1}} f(t) d x_{2} d t d x_{1} \\
& { }_{0} I_{x}^{3} f(x)=\int_{0}^{x} \int_{0}^{x_{1}} f(t) \int_{t}^{x_{1}} d x_{2} d t d x_{1} \\
& { }_{0} I_{x}^{3} f(x)=\int_{0}^{x} \int_{0}^{x_{1}} f(t)\left[x_{2}\right]_{t}^{x_{1}} d t d x_{1} \\
& { }_{0} I_{x}^{3} f(x)=\int_{0}^{x} \int_{0}^{x_{1}} f(t)\left[x_{1}-t\right] d t d x_{1}
\end{aligned}
$$

y cambiando el orden de integración como se muestra se tiene:

$$
\begin{aligned}
& { }_{0} I_{x}^{3} f(x)=\int_{0}^{x} \int_{t}^{x} f(t)\left[x_{1}-t\right] d x_{1} d t \\
& { }_{0} I_{x}^{3} f(x)=\int_{0}^{x} f(t) \int_{t}^{x}\left[x_{1}-t\right] d x_{1} d t \\
& { }_{0} I_{x}^{3} f(x)=\int_{0}^{x} f(t)\left[\frac{\left(x_{1}-t\right)^{2}}{2}\right]_{t}^{x} d t \\
& { }_{0} I_{x}^{3} f(x)=\int_{0}^{x} f(t)\left[\frac{(x-t)^{2}}{2}\right] d t \\
& { }_{0} I_{x}^{3} f(x)=\frac{1}{1 \cdot 2} \int_{0}^{x} f(t)[x-t]^{2} d t
\end{aligned}
$$

De lo anterior se deduce que para 4 integrales se obtiene:

$$
{ }_{0} I_{x}^{4} f(x)=\frac{1}{1 \cdot 2 \cdot 3} \int_{0}^{x} f(t)[x-t]^{3} d t
$$

y para $\mathrm{n}$ integral

${ }_{0} I_{x}^{n} f(x)=\int_{0}^{x} \int_{0}^{x_{1}} \int_{0}^{x_{2}} \ldots \int_{0}^{x_{n-1}} f(t) d t d x_{n-1} d x_{n-2} \ldots d x_{2} d x_{1}$ se tiene la fórmula:

${ }_{0} I_{x}^{n} f(x)=\frac{1}{\Gamma(n)} \int_{a}^{x}(x-t)^{n-1} f(t) d t, t>a, n \in \mathbb{N}$

Además como el límite inferior de la integral es arbitrario entonces se obtiene:

$$
{ }_{a} I_{x}^{n} f(x)=\frac{1}{\Gamma(n)} \int_{a}^{x}(x-t)^{n-1} f(t) d t, t>a, n \in \mathbb{N}
$$

Supongamos que para $\mathrm{n}=\mathrm{k}$ se cumple (1.1):

$$
{ }_{a}^{k} I_{x} f(x)=\frac{1}{\Gamma(\bar{k})} \int_{a}^{x}(x-t)^{k-1} f(t) d t
$$

Entonces se tiene para $\mathrm{n}=\mathrm{k}+1$ :

$$
{ }_{a} I_{x}^{k+1} f(x)=\int_{a}^{t} \frac{1}{\Gamma(k)} \int_{a}^{s}(s-r)^{k-1} f(r) d r d s
$$

Por el teorema de Fubini

$$
\begin{gathered}
=\frac{1}{\Gamma(\mathrm{k})} \int_{\mathrm{a}}^{\mathrm{t}} \int_{\mathrm{r}}^{\mathrm{t}}(\mathrm{s}-\mathrm{r})^{\mathrm{k}-1} \mathrm{dsf}(\mathrm{r}) \mathrm{dr} \\
=\frac{1}{\Gamma(\mathrm{k})} \int_{\mathrm{a}}^{\mathrm{t}} \frac{\left(t-r^{j^{k}}\right.}{k} f(r) d r \\
=\frac{1}{k \Gamma(k)} \int_{d}^{t}(t-r)^{k} f(r) d r
\end{gathered}
$$

aplicando las propiedades de la función Gamma se obtiene:

$$
=\frac{1}{\Gamma(k+1)} \int_{a}^{t}(t-r)^{k} f(r) d r
$$

La ecuación (1.1) es conocida como la fórmula de Cauchy, y puede extenderse para cualquier $\alpha \in \mathbb{R}^{+}$, en la siguiente definición:

Definición (Operador de integración de orden fraccionaria Riemann-Liouville).

Sea $\alpha \in \mathbb{R}^{+}$. El operador de integración de orden fraccionario, denotado por $I_{x}^{\alpha}$, es del tipo RiemannLiouville de orden $\alpha$ de una función $f \in L^{1}[a ; b]$ si

$$
{ }_{a}^{a} f(x)=\frac{1}{\Gamma(\alpha)} \int_{a}^{x}(x-s)^{a-1} f(s) d s, a \leq x \leq b
$$

Linealidad del operador de integración de orden fraccionario:

Proposición (Linealidad del operador de integración de orden fraccionario) 
Sea $\lambda \in \mathbb{R}$ y $a \in \mathbb{R}^{+}$. Si existe el operador de integración ${ }_{a} I_{x}^{\alpha} f(x)$ y ${ }_{a} I_{x}^{\alpha} g(x)$, entonces se cumplen las siguientes propiedades:

1. ${ }_{a} I_{x}^{\alpha}(f+g)(x)={ }_{a} I_{x}^{\alpha} f(x)+{ }_{a} I_{x}^{\alpha} g(x)$

$\forall \mathrm{f}, \mathrm{g} \in L^{1}[\mathrm{a}, \mathrm{b}]$;

2. ${ }_{a} I_{x}^{\alpha} \lambda f(x)=\lambda{ }_{a} I_{x}^{\alpha} f(x) \forall f \in L_{1}[a, b]$ y $\forall \lambda \in \mathbf{R}$.

\section{Demostración}

1. ${ }_{a} I_{x}^{\alpha}(f+g)(x)=\frac{1}{\Gamma(\alpha)} \int_{x}^{a}(x-t)^{\alpha-1}(f+g)(s) d s$ ${ }_{a} I_{x}^{\alpha}(f+g)(x)=\frac{1}{\Gamma(\alpha)} \int_{x}^{a}(x-t)^{\alpha-1} f(s) d s$ $+\frac{1}{\Gamma(\alpha)} \int_{x}^{a}(x-t)^{\alpha-1} g(s) d s$

Por lo tanto

${ }_{a} I_{x}^{\alpha}(f+g)(x)={ }_{a} I_{x}^{\alpha} f(x)+{ }_{a} I_{x}^{\alpha} g(x)$

2. ${ }_{a} I_{x}^{\alpha} \lambda f(x)=\frac{1}{\Gamma(\alpha)} \int_{a}^{x}(x-t)^{\alpha-1} \lambda f(s) d s$ ${ }_{a} I_{x}^{\alpha} \lambda f(x)=\lambda \frac{1}{\Gamma(\alpha)} \int_{a}^{x}(x-t)^{\alpha-1} f(s) d s$

Por lo tanto

${ }_{a} I_{x}^{\alpha} \lambda f(x)=\lambda{ }_{a} I_{x}^{\alpha} f(x) \forall \lambda \in \mathrm{R}$

Ejemplo (Operador de integral de orden fraccionaria). De la definición de Riemann-Liouville calcular la integral fraccionaria de orden $\alpha=1 / 2$, en la función cuya regla de correspondencia es $f(x)=1$.

\section{Solución}

Por definición (Integral fraccionaria) se tiene:

$$
\begin{aligned}
& { }_{a} I_{x}^{1 / 2} f(x)=\frac{1}{\Gamma(1 / 2)} \int_{a}^{x}(x-s)^{1 / 2-1} f(s) d s \\
& { }_{a} I_{x}^{1 / 2}(1)=\frac{1}{\Gamma(1 / 2)} \int_{a}^{x}(x-s)^{-1 / 2}(1) d s
\end{aligned}
$$

Haciendo cambio de variable:

$$
u=x-s \text {, }
$$

y realizando operaciones tenemos:

$$
\begin{aligned}
& s=x-u \\
& s \rightarrow x \Rightarrow u \rightarrow 0 \\
& s \rightarrow a \Rightarrow u \rightarrow x-a \\
& d s=-d u
\end{aligned}
$$

Reemplazando (1.3) en (1.4) y usando la tabla1 se obtiene:

$$
\begin{aligned}
& I_{a}^{1 / 2}(1)=\frac{1}{\sqrt{\pi}} \int_{x-a}^{0} u^{-1 / 2}(-d u) \\
& I_{a}^{1 / 2}(1)=\frac{1}{\sqrt{\pi}} \int_{0}^{x-a} u^{-1 / 2} d u \\
& { }_{a} I_{x}^{1 / 2}(1)=\frac{1}{\sqrt{\pi}}\left[2 u^{1 / 2}\right]_{0}^{x-a} \\
& { }_{a} I_{x}^{1 / 2}(1)=\frac{2}{\sqrt{\pi}}(x-a)^{1 / 2}
\end{aligned}
$$

Operador de Integración de orden fraccionario de una función potencial:

Proposición (Integral del potencial del operador fraccionario).

Si $f(x)=(x-a)^{\beta}, \beta>0, x>a$ entonces
${ }_{a} I_{x}^{\alpha}(x-a)^{\beta}=\frac{\Gamma(\beta+1)}{\Gamma(\alpha+\beta+1)}(x-a)^{\alpha+\beta}$

\section{Demostración}

Por definición (Integral fraccionaria) se tiene: ${ }_{a} I_{x}^{\alpha}(x-a)^{\beta}=\frac{1}{\Gamma(\alpha)} \int_{a}^{x}(x-s)^{\alpha-1}(s-a)^{\beta} d s$

Con la sustitución $s=a+t(x-a)$ de donde se tiene:

$$
\begin{aligned}
& s-a=t(x-a) \\
& x-s=(x-a)(1-t) \\
& \text { Si } s \rightarrow x \Rightarrow t \rightarrow 1 \\
& S i s \rightarrow a \Rightarrow t \rightarrow 0 \\
& d s=(x-a) d t
\end{aligned}
$$

Reemplazando (1.7) en (1.6) se tiene:

$$
{ }_{a} I_{x}^{\alpha}(x-a)^{\beta}=\frac{1}{\Gamma(\alpha)} \int_{J_{0}^{l}}^{l}[(x-a)(1-t)]^{\alpha-1}[t(x-a)]^{\beta}(x-a) d t
$$$$
{ }_{a} I_{x}^{\alpha}(x-a)^{\beta}=
$$$$
\frac{1}{\Gamma(\alpha)} \int_{0}^{1} t^{(\beta+1)-1}(1-t)^{\alpha-1}(x-a)^{\alpha-1}(x-a)^{\hat{\rho}}(x-a) d t
$$

$$
{ }_{a}^{\alpha} I_{x}^{\alpha}(x-a)^{\beta}=\frac{(x-a)^{\alpha+\beta}}{\Gamma(\alpha)} \int_{0}^{1} t^{(\beta+1)-1}(1-t)^{\alpha-1} d t
$$

Luego por la definición de la función Beta se obtiene:

$$
{ }_{a}^{\alpha} I_{x}^{\alpha}(x-a)^{\beta}=\frac{(x-a)^{\alpha+\beta}}{\Gamma(\alpha)} B(\beta+1, \alpha)
$$

Teniendo en cuenta la propiedad de la función Beta (a)

$$
\begin{aligned}
B(\beta+1, \alpha) & =\frac{\Gamma(\beta+1) \Gamma(\alpha)}{\Gamma(\beta+1+\alpha)} \quad \text { se obtiene: } \\
{ }_{a} I_{x}^{\alpha}(x-a)^{\beta} & =\frac{(x-a)^{\alpha+\beta}}{\Gamma(\alpha)} \frac{\Gamma(\beta+1) \Gamma(\alpha)}{\Gamma(\beta+\alpha+1)} \\
{ }_{a} I_{x}^{\alpha}(x-a)^{\beta} & =\frac{\Gamma(\beta+1)}{\Gamma(\alpha+\beta+1)}(x-a)^{\alpha+\beta}, \\
\alpha, \beta>0, x & >a
\end{aligned}
$$


Ejemplo (Operador de Integración de orden fraccionario de una función potencial)

Calcular la integral fraccionaria de orden $\alpha=1 / 3$ y $a=4$ de $f(x)=(x-4)^{1 / 2}$

Solución

De la proposición (Potencial fraccionario) ${ }_{a} I_{x}^{\alpha}(x-a)^{\beta}=\frac{\Gamma(\beta+1)}{\Gamma(\alpha+\beta+1)}(x-a)^{\alpha+\beta}$,

$\alpha, \beta>0, x>a$

${ }_{4} I_{x}^{1 / 3}(x-4)^{1 / 2}=\frac{\Gamma(1 / 2+1)}{\Gamma(1 / 3+1 / 2+1)}(x-4)^{1 / 3+1 / 2}$

${ }_{4} I_{x}^{1 / 3}(x-4)^{1 / 2}=\frac{\Gamma(3 / 2)}{\Gamma(11 / 6)}(x-4)^{5 / 6}$

Por la propiedad de la Función Gamma y la tabla1 se obtiene:

${ }_{4} I_{x}^{1 / 3}(x-4)^{1 / 2}=\frac{\sqrt{\pi}}{2(0,9407 \ldots)}(x-4)^{5 / 6}$

Operador de Integración de una función logarítmica de orden fraccionario:

Proposición (Integral de orden fraccionario de la función logaritmo)

Si $f(x)=\ln (x-a)$ tal que $x>a$ entonces

$$
{ }_{a}^{\alpha} I_{x}^{\alpha} \ln (x-a)=\frac{(x-a)^{\alpha}}{\Gamma(\alpha+1)}[\ln (x-a)-\gamma-\psi(\alpha+1)]
$$

\section{Demostración}

Por la definición de la integral fraccionaria se tiene:

${ }_{a}^{\alpha} \ln (x-a)=\frac{1}{\Gamma(\alpha)} \int_{a}^{x}(x-s)^{\alpha-1} \ln (s-a) d s$

haciendo un cambio de variable:

$$
s=a+t(x-a)
$$

de donde se obtiene:

$$
\begin{aligned}
& s-a=t(x-a) \\
& x-s=(x-a)(1-t) \\
& \text { Si } s \rightarrow x \Rightarrow t \rightarrow 1 \\
& \text { Si } s \rightarrow a \Rightarrow t \rightarrow 0 \\
& d s=(x-a) d t
\end{aligned}
$$

Reemplazando (1.10) en (1.9) se tiene:

$$
\begin{aligned}
& { }_{a} I_{x}^{\alpha} \ln (x-a)= \\
& \frac{1}{\Gamma(\alpha)} \int_{0}^{1}[(x-a)(1-t)]^{\alpha-1} \ln [t(x-a)](x-a) d t \\
& { }_{a} I_{x}^{\alpha} \ln (x-a)=\frac{(x-a)^{\alpha}}{\Gamma(\alpha)} \int_{0}^{1}(1-t)^{\alpha-1} \ln [t(x-a)] d t \\
& { }_{a} I_{x}^{\alpha} \ln (x-a)= \\
& \frac{(x-a)^{\alpha}}{\Gamma(\alpha)} \int_{0}^{1}(1-t)^{\alpha-1}[\ln t+\ln (x-a)] d t
\end{aligned}
$$

$$
\begin{aligned}
& { }_{a} I_{x}^{\alpha} \ln (x-a)=\frac{(x-a)^{\alpha}}{\Gamma(\alpha)} \ln (x-a) \int_{0}^{1}(1-t)^{\alpha-1} d t+ \\
& \frac{(x-a)^{\alpha}}{\Gamma(\alpha)} \int_{0}^{1}(1-t)^{\alpha-1} \ln t d t
\end{aligned}
$$

desarrollando la primera integral y haciendo cambio de variable en la segunda integral de (1.11): $u=1-t$ tenemos:

$$
\left.\begin{array}{l}
t=1-u \\
\text { Si } t \rightarrow 1 \Rightarrow u \rightarrow 0 \\
\text { Si } t \rightarrow 0 \Rightarrow u \rightarrow 1 \\
d t=-d u
\end{array}\right\}
$$

y reemplazando

${ }_{a} I_{x}^{\alpha} \ln (x-a)=$

$\frac{(x-a)^{\alpha}}{\Gamma(\alpha)} \ln (x-a) \frac{1}{\alpha}+\frac{(x-a)^{\alpha}}{\Gamma(\alpha)} \int_{1}^{0} u^{\alpha-1} \ln (1-u)(-d u)$

${ }_{a} I_{x}^{\alpha} \ln (x-a)=$

$\frac{(x-a)^{\alpha}}{\alpha \Gamma(\alpha)} \ln (x-a)-\frac{(x-a)^{\alpha}}{\Gamma(\alpha)} \int_{0}^{1} u^{\alpha-1} \ln (1-u) d u$

Luego, se sabe que $d\left(1-u^{\alpha}\right)=-\alpha u^{\alpha-1} d u$ de donde $u^{\alpha-1} d u=-\frac{d\left(1-u^{\alpha}\right)}{\alpha}$, y reemplazando en la ecuación anterior se tiene:

${ }_{a} I_{x}^{a} \ln (x-a)=$

$\frac{(x-a)^{\alpha}}{\Gamma(\alpha+1)} \ln (x-a)+\frac{(x-a)^{\alpha}}{\alpha \Gamma(\alpha)} \int_{0}^{1} \ln (1-u) d\left(1-u^{\alpha}\right)$

, integrando por partes: $\int v d w=v w-\int w d v$

${ }_{a} I_{x}^{a} \ln (x-a)=$

$\left.\frac{(x-a)^{a}}{\Gamma(\alpha+1)} \ln (x-a)+\frac{(x-a)^{\alpha}}{\Gamma(\alpha+1)}\left[\left(\left(1-u^{\alpha}\right) \ln (1-u)\right)\right)_{0}^{1}+\int_{0}^{1} \frac{1-u^{\alpha}}{1-u} d u\right]$

${ }_{a} I_{x}^{\alpha} \ln (x-a)=$

$\frac{(x-a)^{\alpha}}{\Gamma(\alpha+1)}\left[\ln (x-a)+\left(\left(1-u^{a}\right) \ln (1-u)\right)_{0}^{1}+\int_{0}^{1} \frac{1-u^{\alpha}}{1-u} d a\right]$

Aplicando la propiedad de la función digamma (c)

${ }_{a} I_{x}^{\alpha} \ln (x-a)=$

$\frac{(x-a)^{\alpha}}{\Gamma(\alpha+1)}[\ln (x-a)-\gamma-\psi(\alpha+1)]$

Ejemplo (Integral del orden fraccionario logarítmica) Calcular la integral fraccionaria de orden $\alpha=\frac{1}{3}$ de $f(x)=\ln (x-2)$ 


\section{Solución}

De la proposición (Integral fraccionario del logaritmo) se tiene:

$$
\begin{aligned}
& { }_{a} I_{x}^{\alpha} \ln (x-a)=\frac{(x-a)^{\alpha}}{\Gamma(\alpha+1)}[\ln (x-a)-\gamma-\psi(\alpha+1)] \\
& { }_{2} I_{x}^{1 / 3} \ln (x-2)= \\
& (x-2)^{1 / 3} \\
& \left.\frac{(x / 3+1)}{\Gamma(1 / 3}(x-2)-\gamma-\psi\left(\frac{1}{3}+1\right)\right]
\end{aligned}
$$

Por las propiedades de la función digamma, de la función gamma, y las tablas 1 y 2 se obtienen:

$$
\begin{aligned}
& { }_{2} I_{x}^{1 / 3} \ln (x-2)= \\
& \frac{(x-2)^{1 / 3}}{\Gamma(4 / 3)}\left[\ln (x-2)-\gamma-\left(\psi\left(\frac{1}{3}\right)+\frac{1}{1 / 3}\right)\right] \\
& { }_{2} I_{x}^{1 / 3} \ln (x-2)= \\
& \frac{(x-2)^{1 / 3}}{\Gamma(4 / 3)}\left[\ln (x-2)-\gamma-\left(-\gamma-\frac{\pi}{2 \sqrt{3}}-\frac{3}{2} \ln 3+\frac{1}{1 / 3}\right)\right] \\
& { }_{2} I_{x}^{1 / 3} \ln (x-2)= \\
& \frac{(x-2)^{1 / 3}}{(0,8930 . . .)}\left[\ln (x-2)+\frac{\pi}{2 \sqrt{3}}+\frac{3}{2} \ln 3-3\right]
\end{aligned}
$$

\section{CONCLUSIONES}

El operador de integración de orden fraccionario es una generalización de la integral ordinaria.

La propiedad de la integral ordinaria, bajo ciertas condiciones, también se cumple en la integral de orden fraccionario.

\section{REFERENCIAS BIBLIOGRÁFICAS}

Arafet, P., Domínguez A. y Chang, F. (2008). Una introducción al cálculo fraccionario. Cuba: Universidad del Oriente.

Bell, W. (1968). Special functions for Scientists and Engineers. D. Van Nostrand Company Ltd.

Bonilla, Kilbas, A., y Trujillo, J. (2003). Cálculo fraccionario y ecuaciones diferenciales fraccionarias. Edit. UNED: Madrid.

Bowers, A, \& Kalton, N. (2010). An introductory course in functional Analysis. France: Springer.

Chaudhry, M. A. \& Zubair, S. M. (2002). On a class of incomplete gamma functions with applications. United States of America: Academic Press, INC

Euler.L. (1738). De Progressionibus Transcentibus, sev Quarum Termini Algebraice Dari Nequeunt.Comment.Acad.Sci.Imperia les Petropolitane 5, 38-57.

Guardales, R. (2004). Investigación y enseñanza de la matemática. Sociedad matemática peruana. Perú: edit. Art. Editores

Levedev, N. (1972). Special functions and their applications. First. London: PrenticeHall, Inc.

Liouville, J. (1832). Memoire sur quelques Quéstion de Géometrie et de Mécanique, et sur un nouveu genre de Calcul pour resudre ces Quéstion.J.Ecole Polytech, 13(21) 1-69.

Miller, K. \&Ross, B. (1993).An introduction to the fractional calculus and fractional differential equations. Wiley and Sons: New York

Ortigueira, Manuel. (2011). Fractional calculus for scientists and engineers. 84th ed. Portugal: Springer.

Purcell, E., Varberg, D. y Rigdon, S. (2007). Cálculo diferencial $e$ integral. $9^{a}$ ed. México: Pearson-Education.

Riemann, B. (1876).Versuch einer allegemeinen auffasung der integration und differentiation. Gesammelte Werke, 331-344.

Samko, S., Kilbas, A. \& Marichev, O. (1993). Fractional integrals and derivatives: theory applications. Gordon and Breach. New York

Santanu, D. (2011). Functional fractional calculus. Second. India: Springer. 